British Journal of Ophthalmology, 1988, 72, 897-899

\title{
Day-case cataract surgery
}

\author{
M T WATTS ${ }^{1}$ AND J L PEARCE ${ }^{2}$ \\ From the ${ }^{1}$ Birmingham and Midland Eye Hospital and the ${ }^{2}$ Alexandra Hospital, Redditch
}

SUMmARY The results of a series of 40 cataract extractions with lens implantation performed on day-case patients under local anaesthesia are reported. The ways in which modern surgical techniques have rendered this a safe procedure are described, and the potential benefits of day-case intraocular surgery are discussed.

Day-case surgery is becoming increasingly widespread in Great Britain, and its cost effectiveness and popularity with patients are well established. ${ }^{1}$ Though common in the United States, intraocular day-case surgery has not found universal favour in this country, ${ }^{2}$ though a series of intracapsular cataract extractions without lens implantation has been reported. ${ }^{34}$ The increased complication rate with intracapsular cataract extraction with overnight stay as compared to a five-day stay has been recorded. ${ }^{5}$ In 1983 the Commission on the Provision of Surgical Services of the Royal College of Surgeons published its Guidelines of day-case surgery ${ }^{6}$ and concluded that, in view of the necessity for supportive services, day-case surgery for intraocular procedures was unlikely to become widespread in the near future, and indeed these predictions were accurate.

We believe that using extracapsular surgery, however, and peribulbar as opposed to retrobulbar local anaesthesia, day-case cataract extraction is a safe procedure. In this report we describe a series of $\mathbf{4 0}$ cataract extractions without any problems attributable to day-case management. The importance of patient selection is stressed, as are the potential economies that the technique may bring.

\section{Material and methods}

Forty-one patients were selected as suitable for daycase surgery after inquiry into their home support

Correspondence to $\mathrm{M} T$ Watts, FRCS, Birmingham and Midland Eye Hospital, Church Street, Birmingham B3 2NS. and general physical and mental wellbeing. The procedure was explained to them as an alternative to our standard practice of overnight stay. All consented willingly, and keratometry and ultrasonic axial length measurement were carried out on them as outpatients. They were admitted at $11 \mathrm{am}$ to the day unit of our hospital for afternoon surgery, signed their consent in the presence of the surgeon, and had any further questions answered.

One hour pre-operatively they were given $10 \mathrm{mg}$ of temazepam orally, and one half-hour later the local anaesthetic was administered. This comprised topical $0.4 \%$ oxybuprocaine drops, and a peribulbar injection of $4-6 \mathrm{ml}$ of $0.75 \%$ bupivacaine, injected through the lower eyelid into the peribulbar space but not entering the muscle cone. A Honan balloon was applied until surgery.

Patients who wore hearing aids were encouraged to retain them during surgery, and music was played if so desired. Drapes included the provision of a tent over the patient's mouth into which oxygen was piped.

All operations were performed by one of two surgeons, one of whom used a standard extracapsular technique, with removal of the anterior capsule prior to delivery of the nucleus, and the other an intercapsular technique, in which the nucleus was delivered through a horizontal cut in the anterior capsule and the anterior capsule removed after the insertion of the lens implant into the capsular bag. Both inserted capsular fixated lenses, of either a disc or Y-loop type. In two of the patients a lens was not inserted as a primary procedure owing to peroperative posterior capsule rupture. Sodium hyaluronate 
was used throughout to protect intraocular structures, but was removed just prior to full closure of the section, which was done using $9 / 0$ or $10 / 0$ nylon. A subconjunctival injection of ampicillin and DepoMedrone (methylprednisolone) was given as well as two drops of timolol $0.5 \%$. An eye pad and plastic cartella were applied. Patients were then taken back to the ward, whence they were collected by friends or relatives shortly after.

They all returned at 9 am the following day for their first dressing, at which time they were examined by the surgeon. All were given Maxitrol (neomycin, polymyxin $\mathrm{B}$, dexamethasone) eye drops to take 2 hourly until their next clinic visit at between two and five days, when they again had a full ocular examination with tonometry. Subsequent visits were arranged as required, usually being at six weeks postoperatively, at which time they were referred to an optician for provision of spectacles.

\section{Results}

Forty cataract operations were performed in a group of 22 males and 19 females, with a mean age of $74 \cdot 4$ years, range 62-91. Twenty-six of the patients were in excellent general health, whereas the others suffered from the conditions shown in Table 1. All were able to lie still and flat during surgery. Their social circumstances varied, but all had either a relative or friend to stay with them over the first postoperative night except for one who lived in warden controlled accommodation and was closely supervised, and one for whom district nurse visiting was arranged.

Fourteen had had previous cataract surgery, but none as a day case. Only one patient required to be admitted overnight, and this was due to adverse weather preventing his collection from hospital. One patient was so anxious on admission that she decided to decline surgery and was discharged. She was subsequently offered an operation under general anaesthesia, but remained undecided as to whether to proceed.

None of the patients suffered any medical problems during their stay. In two patients no intraocular lens was inserted owing to posterior capsule rupture, though implantation as a secondary procedure is now planned. In neither did the surgeon consider that the use of day-case care had compromised the operation, and the anaesthesia had been effective.

There were two instances of a rise in intraocular pressure above $21 \mathrm{mmHg}$, to $24 \mathrm{~mm}$ and $27 \mathrm{~mm}$ respectively. These were transient and did not require treatment. There was no case of iris prolapse or endophthalmitis.

Patient satisfaction, though difficult to quantitate,
Table 1 Systemic illnesses suffered by day-case patients

\begin{tabular}{ll}
\hline Illness & No. \\
\hline Bronchitis/emphysema & 3 \\
Diabetes mellitus (oral control) & 2 \\
Previous cerebrovascular accident & 2 \\
Arthritis & 3 \\
Hypertension & 6 \\
\hline
\end{tabular}

was generally good. Only one patient regretted her decision to have day-case surgery rather than general anaesthesia, and six patients have requested surgery to their second eye on a similar basis.

\section{Discussion}

Much of the concern regarding day-case cataract surgery rests on the fear of raised intraocular pressure, iris prolapse, and endophthalmitis going unnoticed for a period of time. We believe that our techniques of anaesthesia and surgery minimise these problems, and in this series we have so far experienced none of them.

Posterior peribulbar anaesthesia, first described by Davis and Mandel, ${ }^{7}$ though previously performed for many years, reduces the risks normally associated with retrobulbar anaesthesia. Because the anaesthetic is not injected within the muscle cone, the risk of damage is much lessened - in particular the risks of retrobulbar haemorrhage, optic nerve damage, perforation of the globe, and meningeal irritation or respiratory depression. It provides good analgesia and akinesia, and obviates the need for intravenous sedatives or neuroleptics and hence the need for the presence of an anaesthetist, though in all cases emergency anaesthetic cover was available.

Extracapsular cataract surgery with capsular fixated lenses, and removal of sodium hyaluronate prior to full wound closure reduces the incidence of postoperative pressure rises that others performing day-case or short-stay cataract surgery have experienced when using intracapsular surgery with chymotrypsin. ${ }^{5}$ This, in combination with meticulous attention to wound closure, we believe is responsible for the lack of iris prolapse. In a number of patients in the series $9 / 0$ rather than $10 / 0$ nylon was used as suture material in order to provide added wound strength. With buried knots these did not prove uncomfortable to patients nor did they cause any astigmatic problems. This may be a more appropriate suture for day-case patients. Subconjunctival antibiotics were routinely used to minimise risks of infection.

Apart from patient preference the cost savings made by day-case surgery are significant. Precise 
estimates of the savings are difficult to formulate, since as long as facilities exist for inpatient management their disuse does not offer immediate savings. In our hospital we have fully utilised both the inpatient and outpatient day-case facilities, so the effect has been to reduce the waiting list rather than to effect immediate savings. Estimates of savings in the region of $£ 270$ have recently been suggested, however.

Day-case cataract surgery is not suitable for all patients, and selection is critical. We do not see the procedure as a replacement for conventional inpatient management but rather as an adjunct to it. Particularly in times of ever increasing demands on Health Service funds, intraocular day-case surgery helps to make the best of resources.

\section{References}

$1 \mathrm{Ogg}$ TW, Obey PA. The workload of a purpose built day surgical unit. Ann R Coll Surg 1987; 69: 110-2.

2 Anonymous. Day-case cataract surgery. Lancet 1983; ii: $383-4$.

3 Ingram RM. Day-case ophthalmic surgery. (Editorial.) $J R$ Soc Med 1984; 77: 263-5.

4 Ingram RM, Banerjee D, Traynar MJ, Thompson RK. Day-case cataract surgery. Br J Ophthalmol 1983; 67: 278-81.

5 Vernon SA, Cheng H. Comparison between the complications of cataract surgery following local anaesthesia with short stay and general anaesthesia with a five day hospitalisation. $\mathrm{Br} J$ Ophthalmol 1985; 69: 360-3.

6 Commission on the provision of surgical services. Guidelines for day-case surgery. Royal College of Surgeons of England, 1985.

7 Davis DB, Mandel MR. Posterior peribulbar anaesthesia: an alternative to retrobulbar anaesthesia. J Cataract Refractive Surg 1986; 12: $182-4$.

Accepted for publication 10 September 1987. 